\title{
THE EFFECT OF AGING AND GROUND TEXTURE ON THE CONTROL OF BRAKING
}

\author{
Zheng Bian \& George J. Andersen \\ Department of Psychology, University of California, Riverside \\ Riverside, California, USA \\ Email: andersen@ucr.edu
}

\begin{abstract}
Summary: In the current study we examined age-related difference in the use of visual information in regulating braking. Younger and older drivers were presented with computer generated 3-D scenes simulating driving on a roadway towards three red stop signs at a constant speed. The task of the drivers was to control braking and to stop as close as possible to the stop signs. The texture density on the ground, initial time to contact (TTC) and initial distance from the stop signs were manipulated. We found that older drivers had larger mean stop distance and lower crash rate than younger drivers. In addition, older drivers, as compared to younger drivers, tended to regulate $\dot{t}$ more frequently at values larger than -0.5 and less frequently at values smaller than -0.5 . These results, taken together, suggest that older drivers may use a more conservative strategy to control braking in order to avoid collisions.
\end{abstract}

\section{INTRODUCTION}

An important driving task is to successfully detect and avoid impending collisions with other moving vehicles, pedestrians or stationary obstacles. When a driver approaches a stop sign or a red light the driver needs to apply appropriate pressure on the brake at the appropriate time.

An important issue is what kind of visual information is used to regulate and control braking. As a driver approaches an object, the projection of the object expands. Lee (1976) showed that the time to contact $(\tau)$ with an approaching object is determined by the visual angle and the inverse rate of optical expansion of that object. Furthermore, Lee (1976) proposed that the time derivative of $\tau$ (tau-dot, or $\dot{\tau}$ ) could be used to regulate deceleration during braking. Maintaining $\dot{\tau}$ at a critical value of -0.5 would lead to a perfect stop. $\dot{t}$ less or greater than -0.5 would result in a collision or stopping before the ideal stop location, respectively. Thus, a possible strategy to control braking is by regulating $i$ at the critical value of -0.5 .

Previous studies have examined whether drivers could use $\dot{t}$ to discriminate collisions and regulate braking. For instance, Kim et. al. (1993) presented drivers with displays simulating approaching a square and had them determine whether the impending collision was hard or soft. They found that when deceleration was manipulated such that $\dot{t}$ was maintained at a constant value, drivers tended to use $\dot{t}$ of -0.5 to discriminate between hard and soft collisions. Yilmaz and Warren (1995) examined the use of $i$ in active control of braking and found that drivers regulated deceleration to null the error between the current $\dot{\tau}$ and the critical $\dot{\tau}$ of -0.5 . If $\dot{\tau}$ is the only source of visual information used in braking control, then manipulating other sources of visual information should not affect the performance in judging collision events. Andersen et. al. (1999) tested this hypothesis using a collision judgment task. Drivers viewed scenes simulating approaching an object with constant deceleration and determined whether they would crash into 
the object. They found that drivers' performance was correlated with $\dot{\tau}$. However, performance was also affected by the initial speed of the driver, size of the impending object and the edge rate on the ground. They suggested that since the critical value of $i$ at -0.5 was the only value at which deceleration was constant, drivers might have used an analysis based on constant deceleration rather than constant $\dot{t}$. Using an active braking control task similar to that in Yilmaz and Warren (1995), Fajen (2005) found that both the global optical flow information and edge rate affected performance in braking, which could not be explained by an analysis based solely on $\dot{t}$. Fajen (2005) proposed that drivers maintained a constant deceleration under the constraint of the maximum possible deceleration. A similar analysis based on a deceleration strategy was examined by Rock, Harris and Yates (2006) in explaining braking performance during realworld driving.

In the current study, we examined whether there was an age-related difference in the use of visual information to regulate braking. Andersen et al. (2000) found that older drivers were less sensitive to collision events as compared to younger drivers, especially at high speed. In addition, older drivers made more collision judgments than younger drivers when non-collision events were simulated. Their findings suggest that older drivers may be at higher risk in detecting collisions during deceleration. In the current study, we examined whether there was an agerelated difference in active control of braking and whether such difference was related to differential use of visual information from ground texture.

\section{EXPERIMENT}

\section{Methods}

Drivers. The drivers were 12 undergraduate students (6 males and 6 females, mean age $=21.2 \pm$ 2.4) and 12 older drivers (6 male and 6 female, age $=71.6 \pm 4.4$ ) from the Life Society Program at the university. All drivers were paid for their participation and were screened using several perceptual and cognitive tests, including Snellen acuity, contrast sensitivity, forward and backward digit span, perceptual encoding, Useful Field of View, and the Kaufman Brief Intelligence Test. In addition, all drivers had normal or corrected-to-normal vision, had a minimum of 2 years of driving experience, and were naïve to the purpose of the study.

Apparatus. The displays were presented on a 58-inch $(148 \mathrm{~cm}$ ) flat screen plasma TV (Panasonic TH-58PF12) with a pixel resolution of $1920 \times 1080$, controlled by a Windows 7 Professional Operating System on a Dell Precision T7500 workstation. The refresh rate was $60 \mathrm{~Hz}$. The dimensions of the display on the monitor were $128.4 \mathrm{~cm}(\mathrm{~W}) \times 72.2 \mathrm{~cm}(\mathrm{H})$, subtending a visual angle of $106.4^{\circ} \times 73.9^{\circ}$ at a viewing distance of $48 \mathrm{~cm}$. A Logitech G25 steering system with acceleration and brake pedals was used for closed loop control of the simulator.

Stimuli. The stimuli were computer generated 3-D scenes simulating driving on a roadway towards three red octagon-shaped stop signs. The roadway had a width of 8 meters, demarked by dashed lines ( $4 \mathrm{~m}$ in length and $0.1 \mathrm{~m}$ in width positioned every 12 meters along the roadway). One stop sign was located in the center of the roadway and the other two were positioned on both sides. Each side of the stop sign was $0.2 \mathrm{~m}$, and the distance between stop signs was $0.6 \mathrm{~m}$. Each stop sign was connected to the ground surface by a red pole with a width of $0.1 \mathrm{~m}$ and a height of 


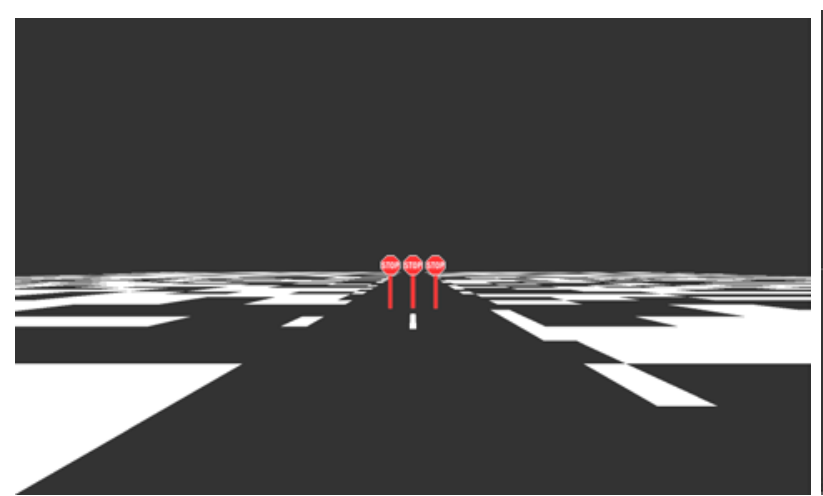

a.

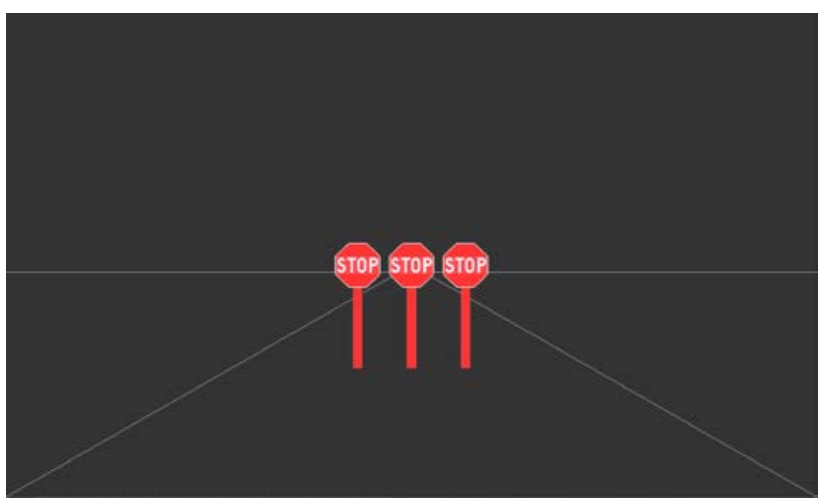

b.

Figure 1. Static image of driving scenes used in the present study. (a) medium texture density on the ground; (b) no texture on the ground

$0.94 \mathrm{~m}$. The center of the stop signs was $1.2 \mathrm{~m}$ above the ground, which was the same as the simulated eye height. The ground surface on both sides of the roadway had either black and white checkerboard texture at different density or no texture. In the no texture condition, only the roadway, the horizon, and the stop signs were visible (Figure 1).

Design. The four-way mixed design included age as the between-subject variable and three within-subject variables: (1) the texture density on the ground (no texture, low density, medium density, or high density), (2) initial TTC (3s, 3.5s, or 4s), and (3) initial distance from the stop signs $(40 \mathrm{~m}, 45 \mathrm{~m}$, or $50 \mathrm{~m})$. The texture density was blocked and the order of texture density was counterbalanced across drivers within each age group. The mean stop distance to the stop signs, crash rate, and distribution of $i$ were collected and analyzed.

Procedure. The experiment was run in a darkened room. Each trial started with a preview phase simulating the drivers moving on a roadway towards three stop signs at a constant speed determined by the initial TTC and initial distance. The drivers did not have control over the brake during the preview phase. Ten seconds later, drivers heard a warning tone which indicated that the control input from the drivers was allowed. The task of the drivers was to control the brake to stop as close as possible to the stop signs. Drivers were instructed to apply smooth and continuous braking as if they were driving a real vehicle. They were also instructed to avoid sudden and jerky brake adjustments or applying full brake pressure at the very last moment (Yilmaz \& Warren, 1995; Fajen, 2005).

The experiment contained 10 blocks. The first two blocks were practice blocks with 72 trials in each block. These served to familiarize the drivers with the control dynamics of the brake and the task. The practice blocks were followed by eight experimental blocks, two for each level of texture density. In each block, each of the 9 combinations of initial TTC and initial distance was repeated 6 times. In addition, 9 practice trials were inserted at the beginning of each block. A break was given after each block. The duration of each block was approximately 45 minutes.

\section{Results}

Similar to Yilmaz and Warren (1995), data analysis excluded trials in which drivers showed slam-on-the-brake or bang-bang patterns. Since two older drivers showed slam-on-the-brake 
pattern in majority of the trials ( $>75 \%)$, their data were not included in analysis. In the remaining drivers, $13.1 \%$ of the total trials were excluded from analysis, which was in line with the $17 \%$ exclusion rate in Yilmaz and Warren (1995). On average, the exclusion rate was 11.1\% for younger drivers and $15.4 \%$ for older drivers. However, this difference did not reach significant $(\mathrm{F}(1,20)=1.16, \mathrm{p}=.30)$. Mean stop distance, and crash rate were collected and analyzed in a 2 (age) $\times 4$ (texture density) $\times 3$ (TTC) $\times 3$ (initial distance) mixed ANOVA. All $\dot{\tau}$ values were collected and categorized into 10 different bins between -1.0 to 0 and then analyzed in a 2 (age) $\times 4$ (texture density) $\times 10$ (bin) mixed ANOVA.

Mean stop distance. There was a significant main effect of age $(F(1,20)=14.18, p<.01)$. The average stop distance for younger and older drivers was $1.0 \mathrm{~m}$ and $2.9 \mathrm{~m}$ from the stop signs, respectively. There was also a significant main effect of initial TTC $(\mathrm{F}(2,40)=9.86, \mathrm{p}<.01)$, a significant main effect of initial distance $(F(2,40)=10.05, \mathrm{p}<.01)$, a significant interaction between TTC and initial distance $(\mathrm{F}(4,80)=5.73$, $\mathrm{p}<.01)$, and a significant interaction between texture density and initial distance $(F(6,120)=2.23, \mathrm{p}<.05)$. According to these results, mean stop distance decreased with increasing initial distance, especially when there was no texture on the ground (see Figure 2). In addition, the mean stop distance increased with increased initial TTC, particularly at short initial distance. No other main effect or interaction was significant.

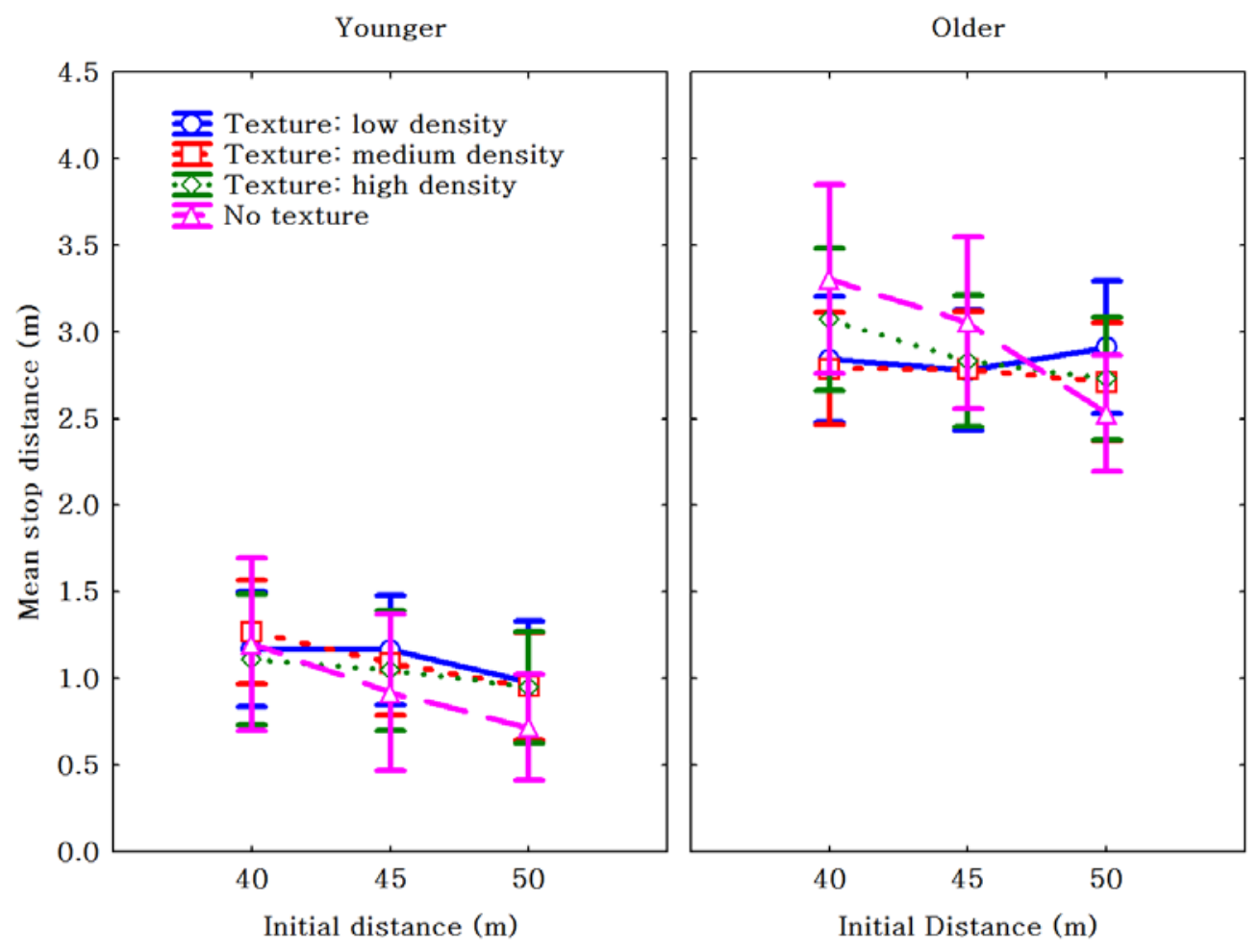

Figure 2. Mean stop distance as a function of age, initial distance and ground texture (Error bars represent \pm 1 standard error)

Crash rate. There was a significant main effect of age $(\mathrm{F}(1,20)=6.76, \mathrm{p}<.01)$. Older drivers had a crash rate of $3 \%$, which was lower than younger drivers' crash rate of $9 \%$. There was a significant main effect of texture density $(\mathrm{F}(3,60)=4.60$, $\mathrm{p}<.01)$, initial TTC $(\mathrm{F}(2,40)=21.84$, 

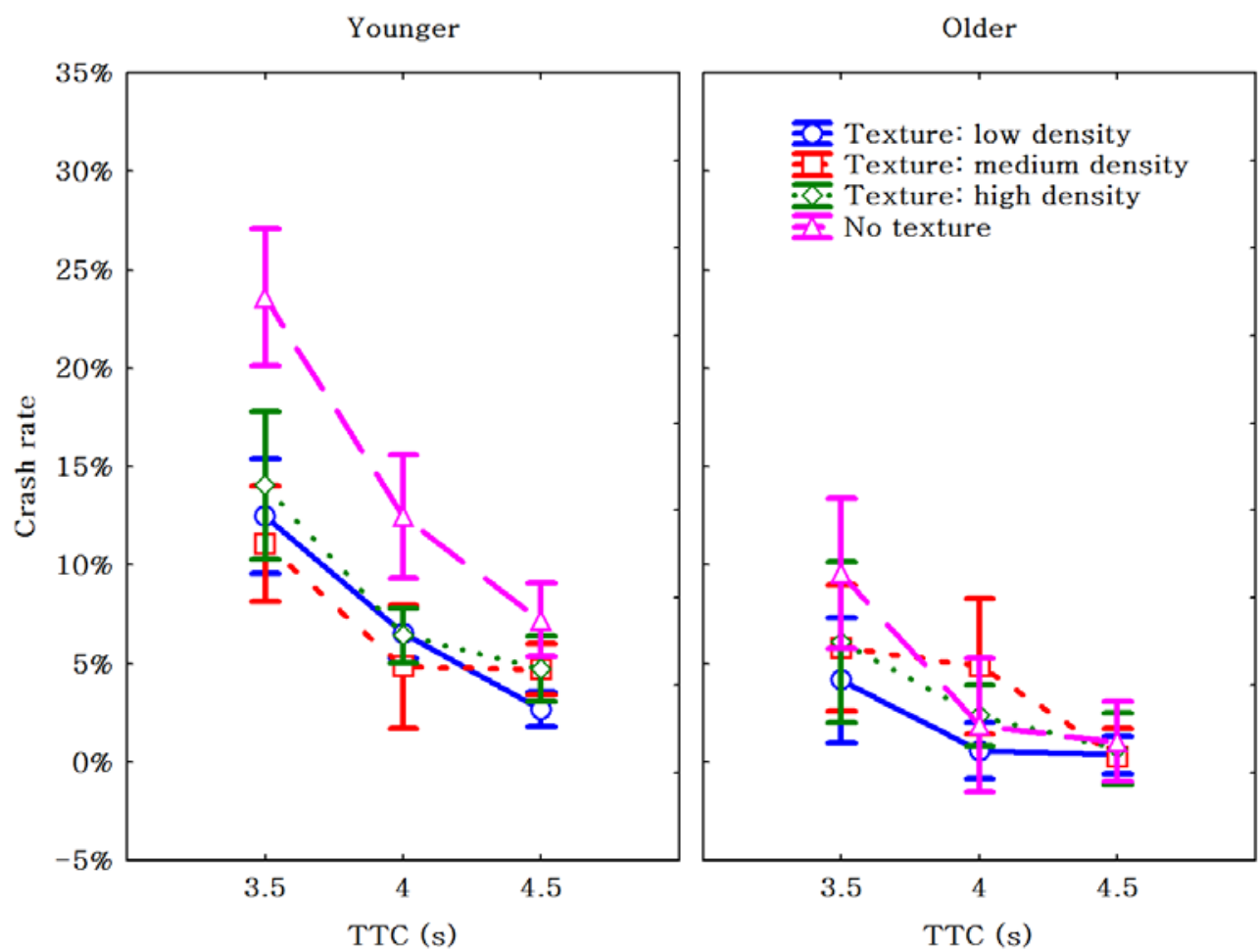

Figure 3. Crash rate as a function of age, ground texture, and initial TTC (Error bars represent \pm 1 standard error)

$\mathrm{p}<.01)$, and initial distance $(\mathrm{F}(2,40)=18.45, \mathrm{p}<.01)$. In addition, there was also a significant interaction between texture density and initial distance $(\mathrm{F}(6,120)=2.57, \mathrm{p}<.05)$, between TTC and initial distance $((\mathrm{F}(4,80)=4.54, \mathrm{p}<.01)$, and a significant 3-way interaction between texture density, TTC, and initial distance $(\mathrm{F}(12,240)=1.97, \mathrm{p}<.05)$. As can be seen from Figure 3, crash rate decreased with longer TTC. When there was no texture on the ground, the crash rate was higher than when there was texture on the ground, suggesting the importance of ground texture on braking control. No other main effect or interaction was significant.

Distribution of $\dot{\tau}$. To analyze the use of $\dot{\tau}$ we determined the proportion of braking time for different values of $i$ specified in 10 different equal interval bins that ranged from -0.9 to 0.0 . There was no significant main effect of age $(F(1,20)<1)$ or ground texture $(F(3,60)<1)$. However, there was a significant main effect of bin $(F(9,180)=25.37, p<.01)$ and a significant interaction between age and bin $(F(9,180)=2.32, \mathrm{p}<.05)$. Figure 4 demonstrates the proportion of $\dot{t}$ at each level of bins for both age groups. As can be seen, younger drivers have the highest proportion of $i(15.6 \%)$ at -0.5 , which is consistent with previous studies providing support to regulating $i$ at -0.5 in collision judgments (Andersen et. al., 1999, 2000; Andersen \& Sauer, 2004) and active braking control (Andersen \& Sauer, 2004; Yilmaz \& Warren, 1995; Fajen, 2005). Older drivers, however, have the highest proportion (14.0\%) of $i$ at -0.4 . Compared to younger drivers, older drivers tend to regulate $\dot{t}$ more frequently at values greater than -0.5 and less frequently at values less than -0.5 . Since $\dot{t}$ less than -0.5 would result in collision, this result suggests that older drivers are more conservative than younger drivers in regulating braking. No other main effect or interaction was significant. 


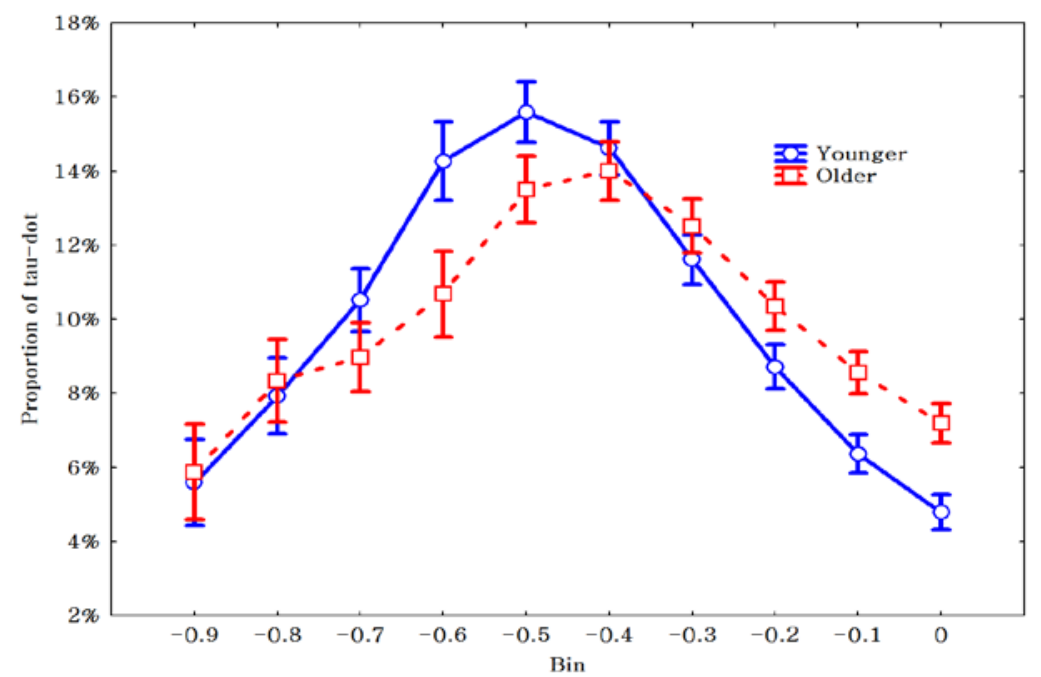

Figure 4. Distribution of $i$ for older and younger drivers (Error bars represent \pm 1 standard error)

\section{DISCUSSION}

In this study, we examined whether there was an age-related difference in braking control and whether such difference was related to the use of visual information. We found that overall, older drivers tend to stop further away from the stop signs and had lower crash rate as compared to younger drivers. Since the drivers were instructed to stop as close to the stop signs as possible, our results show that older drivers, as compared to younger drivers, tend to make safer stops that were further away from the stop signs. In addition, older drivers regulated deceleration such that they had higher proportion of $\dot{t}$ at larger values and lower proportion of $\dot{t}$ at smaller values than younger drivers. Previous studies have found that younger drivers regulated $t$ at -0.5 in braking control (e.g. Yilmaz \& Warren, 1995), suggesting that younger drivers used either a $\dot{t}$ based analysis or constant deceleration based analysis. Our results showed that older drivers may regulate $\dot{t}$ slightly greater than -0.5 , suggesting that they may be cognizant of their decrements in perceptual processing and thus using a strategy not completely based on constant deceleration or $i$ in order to avoid collisions.

In the current study the texture density on the ground was manipulated. There were two purposes for this manipulation. First, texture density is directly related to the edge rate information, which has been shown to affect perceived speed of locomotion (Larish \& Flach, 1990). Previous studies have found that edge rate affected performance in collision judgments (Andersen et. al., 1999) and active braking control (Fajen, 2005). In the current study we examined whether manipulation of texture density affected distribution of $\dot{\tau}$. Although texture density affected mean stop distance and crash rate, we did not find any significant effect of texture density on the regulation of $i$. Second, recent studies have found age-related difference in using the information on the ground surface to organize layout of 3-D scenes (Bian \& Andersen, 2008) and age-related difference in perceiving egocentric distance (Bian \& Andersen, in press). According to the constant deceleration model proposed by Andersen et. al. (1999), judgment of collision events during deceleration was determined not only by $\dot{t}$ but also by the perceived distance between the driver and the object. Thus, it is possible that the manipulation of texture density would have a differential effect on performance in active braking control between younger and older drivers. 
However, the results showed that the effect of texture density did not vary as a function of age. One possible explanation is that perceived distance is not used in active control of braking. On the other hand, it is possible that egocentric distance perception is affected by global optical flow information in self-motion. Future research is needed to examine this issue.

In conclusion, the results of the current study indicate that older drivers had greater stop distances and lower crash rates than younger drivers in active control of braking. While younger drivers used an analysis of $i$ or constant deceleration to regulate braking, older drivers may have used a different strategy in order to reduce crash rate and increase driving safety.

\section{ACKNOWLEDGMENT}

This research was supported by NIH AG031941 and EY18334.

\section{REFERENCES}

Andersen, G. J., \& Sauer, C. W. (2004). Optical information for collision detection during deceleration. In H. Hecht \& G. J. P. Savelsbergh (Eds.) Time to contact: Advances in psychology (Vol. 135, pp. 93-108). Amsterdam: Elsevier.

Andersen, G. J., Cisneros, J., Atchley, P., \& Saidpour, A. (1999). Speed, size, and edge-rate information for the detection of collision events. Journal of Experimental Psychology: Human Perception and Performance, 25, 256-269.

Andersen, G. J., Cisneros, J., Saidpour, A. \& Atchley, P. (2000). Age-related differences in collision detection during deceleration. Psychology and Aging, 15, 241-252.

Bian, Z. \& Andersen, G. J. (in press) Aging and the perception of egocentric distance. Psychology and Aging.

Bian, Z., \& Andersen, G. J. (2008). Aging and the perceptual organization of 3-D scenes. Psychology and Aging. 23(2), 342 - 352.

Fajen, B. R. (2005). Callibration, information, and control strategies for braking to avoid a collision. Journal of Experimental Psychology: Human Perception \& Performance, 31, $480-501$.

Kim, N.-G., Turvey, M. T., \& Carello, V. (1993). Optical information about the severity of upcoming contacts. Journal of Experimental Psychology: Human Perception and Performance, 19, 179-193.

Larish, J. F., \& Flach, J. M. (1990). Sources of optical information useful for perception of speed of rectilinear self-motion. Journal of Experimental Psychology: Human Perception and Performance, 16, 295-302.

Lee, D. N. (1976). A theory of visual control of braking based on information about time-tocollision. Perception, 5, 437-459.

Rock, P. B., Harris, M. G. \& Yate, T. (2006). A test of the tau-dot hypothesis of braking control in the real world. Journal of Experimental Psychology: Human Perception and Performance, 32(6), $1479-1484$.

Yilmaz, E. H., \& Warren, W. H. (1995). Visual control of braking: A test of the $\dot{t}$ hypothesis. Journal of Experimental Psychology: Human Perception and Performance, 21, 996-1014. 